\title{
A novel demonstration of induced EMF
}

William Lonc

Citation: American Journal of Physics 61, 90 (1993); doi: 10.1119/1.17392

View online: http://dx.doi.org/10.1119/1.17392

View Table of Contents: http://scitation.aip.org/content/aapt/journal/ajp/61/1 ?ver=pdfcov

Published by the American Association of Physics Teachers

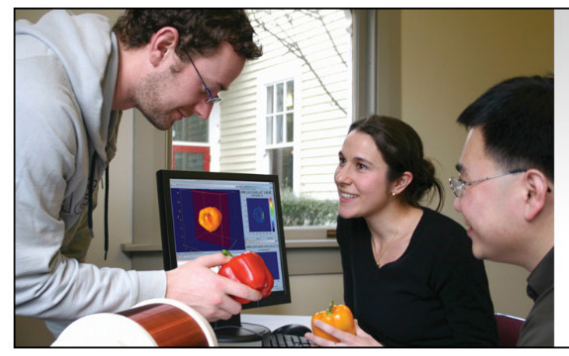

\section{Teach NMR and MRI}

Hands-on education with Terranova-MRI Includes complete student guide with 12 experiments and online videos. 
James L. Hunt, Editor

University of Guelph, Guelph, Ontario NIG 2WI, Canada

This department welcomes brief communications reporting new demonstrations, laboratory equipment, techniques, or materials of interest to teachers of physics. Notes on new applications of older apparatus, measurements supplementing data supplied by manufacturers, information which, while not new, is not generally known, procurement information, and news about apparatus under development may be suitable for publication in this section. Neither the American Journal of Physics nor the Editors assume responsibility for the correctness of the information presented. Submit materials to James L. Hunt, Editor.

\title{
A novel demonstration of induced EMF
}

\author{
William Lonc \\ Physics Department, Saint Mary's University, Halifax, N.S., B3H 3C3 Canada
}

(Received 19 May 1992; accepted 22 June 1992)

In the course of trying to find a somewhat fresh demonstration of induced EMF for an algebra-based physics class, it occurred to us that a simple battery-powered radio could be a good candidate. The hope was that the radio is a much more familiar object to the students than are the various coils and meters that are usually used to demonstrate the effect. The plan was to hold the radio-tuned to a local station in the AM (amplitude modulation) broadcast band ( 550 to $1600 \mathrm{kHz}$ )-in front of the class and then to rotate the radio and demonstrate that there was an orientation at which the signal was maximum and another orientation at which the signal was minimum (perhaps even zero!). It was known that the electric field of the AM transmissions is vertically polarized so that the magnetic field would be horizontally polarized. Hence, the built-in antenna (a solenoid!) could play the role of the detector coil in an induced EMF demonstration.

Inspection of several abandoned radios revealed that they all had "automatic volume control" (AVC) circuits that adjust the overall gain of the radio to maintain a fairly constant audio output (for a given setting of the volume control). This feature was perceived as a major obstacle for the proposed demonstration because the difference in sound level between maximum and minimum induced EMF orientations would not be all that dramatic, especially if a local station were being used as the source of the oscillating magnetic field. Manufacturers, unfortunately, do not foresee the possibility that a user might wish to disable the AVC-in other words, there was no switch with which the AVC could be disabled.

Since the radios were old and abandoned, it was decided to disable the AVC by appropriate modifications to the circuit. Hence, a radio (Radio Shack No. 12-108) that was accompanied by a circuit diagram-here the manufacturer deserves plaudits - was inspected, and it was readily found that cutting one wire and soldering it to another point in the circuit would implement the required modification. Figure 1 shows a partial schematic diagram of the relevant section of the radio. On the basis of our experience, this version of an AVC circuit is probably the most common form. A cut is made at point " $X$," and the free end of R6 is then soldered to ground. This converts the radio into a fixed gain receiver-for our purposes, equivalent to a highly sensitive voltmeter connected to a solenoid (the antenna coil). As part of the demonstration, the back of the case is removed, and the class is shown the coil.

Some words of caution. First, some radios have very complicated AVC circuits and are not worth the trouble needed to disable the AVC. Second, note that the FM band is not suitable because of the way that FM works as well as the possible presence of an AGC (automatic gain control). Moreover, the FM wave is much more prone to depolarization effects. Third, it is true that communications radios usually have a switch to control the AVC, but they usually do not have a loop antenna-and it is the loop antenna that is the "heart" of this demonstration.

At the cost of a few minutes of time, the instructor is equipped with a relatively dramatic piece of apparatus with which to demonstrate induced EMF. The audience hears its favorite music changing drastically in intensity as it watches a familiar device being rotated in front of their eyes.

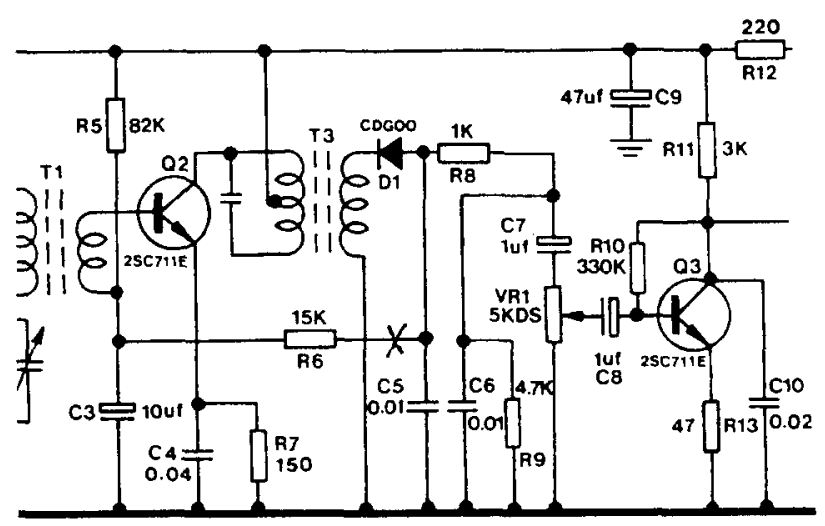

Fig. 1. Partial circuit diagram of a radio equipped with automic volume control (AVC). In normal usage, the negative voltage appearing at " $\mathrm{X}$ " depends on signal strength at the antenna and controls the bias-and thus the gain-of transistor Q2. Disconnecting R6 at " $\mathrm{X}$ " and connecting it to ground removes AVC action. 